\title{
Late-onset depression: issues affecting clinical care
}

\author{
Hannele Variend \& Y. Vishnu Gopal
}

\begin{abstract}
A growing body of evidence suggests that late-onset depression (depression occurring for the first time in later life) differs from early-onset (recurrent) depression in terms of clinical features, aetiology, neuroanatomical substrates and prognosis. Some evidence suggests that late-onset depression is more associated with somatic symptoms, cognitive deficits, cerebral structural abnormalities, vascular disease ('vascular depression') and poorer treatment outcomes than is early-onset depression. Both general adult and old age psychiatrists face the challenges of managing late-onset depression. In this article we attempt to clarify specific issues relating to late-onset depression in terms of clinical features, aetiology, treatment response and prognosis.
\end{abstract}

There is some scepticism regarding the divided classification of depression on the grounds of age at onset, primarily owing to a lack of valid methods for pinpointing that age. Some believe it futile to distinguish between early- and late-onset depression even if there may be evidence that they are different. However, late-onset depression is recognised to be aetiologically different from depression of early onset, and aetiologically heterogeneous in itself.

There is no consensus as to what age should distinguish between the late- and early-onset disorders. In the UK, 60 years of age is the most commonly used cut off, whereas in the USA and other countries 50 is generally used (Baldwin \& $\mathrm{O}^{\prime}$ Brien, 2002). Hopkinson (1964) found that the prevalence of depressive disorders in first-degree relatives of individuals with late-onset depression (aged over 55 at onset), being less than half that for probands with early-onset depression, substantiates Caine et al's (1994) theory that familial risk in affective disorders reduces after 50 years of age. Hence, it is likely that a significant number of patients with lateonset depression in the UK are seen by general adult psychiatrists, who therefore need to be as aware of the specific challenges this disorder poses in terms of aetiology, management and treatment as are old age psychiatrists.

The importance of clinical differentiation between late-onset and early-onset depression is illustrated by findings that late-onset depression may be associated with greater incidence of completed suicide (Bellini \&
Matteucci, 2001) and a poorer outcome (Baldwin et al, 2004) than early-onset depression. Poorer outcomes appear to be related to neuropsychological deficits (Lockwood et al, 2000), which appear to occur more frequently in those who first experience depression after 60 years of age (Holroyd \& Duryee, 1997).

We carried out an extensive search of published literature on electronic databases, including PubMed and Datastar (Medline, Embase, PsychINFO, CINAHL, AMED, King's Fund, DH-Data and DNI), and electronic journals (ProQuest), using various search terms, including 'late-onset depression'. We also reviewed literature identified from a hand search of peer-reviewed journals and cross-references. Studies used various ages as cut-off points for diagnosing late-onset depression, and we have quoted these where relevant.

\section{Epidemiology}

In the population aged over 65 the overall prevalence of major depression is estimated at $2 \%$ and the prevalence of depressive symptoms $10-15 \%$. This figure is increased in hospital sub-populations, and higher still in residential homes (Fountoulakis et al, 2003). The multicentre ODIN study (Ayuso-Mateos et al, 2001), which looked at prevalence of depression in five European countries, classified patients into three age groups: $18-25,26-45$ and 46-65. They found no significant difference in prevalence between the under- 45 and the over- 45 age groups.

\footnotetext{
Hannele Variend is an academic specialist registrar in general adult psychiatry working in the Yorkshire Deanery. Y. Vishnu Gopal is a consultant psychiatrist at the Hartington Unit (Royal Hospital, Chesterfield S44 5BL, UK. Email: vgopal@doctors.org.uk). His interests include assessment and management of cognitive dysfunctions.
} 
We found no study that throws light on the incidence or prevalence of depression in the different 'age at onset' groups. However, in a study of 74 out-patients with depression, Holroyd \& Duryee (1997) found that 59 (almost 80\%) had onset of depression after age 60. Baldwin \& O’Brien (2002) state that as many as three-quarters of patients with major depressive disorder presenting to old age psychiatrists have late-onset depression.

\section{Clinical features}

Table 1 summarises the key clinical differences between early- and late-onset depression reported in various studies.

\section{Depressed mood and cognitions}

Depressed mood, the cardinal symptom of depression, may be less prominent in depression affecting patients in old age (Gurland, 1976), meaning that recognition of late-onset depression in its early stages can be difficult for patients, carers and clinicians. In addition, elderly people with new or recurrent depression are more hypochondriacal and more delusional than younger people, possibly complicating diagnosis (Gurland, 1976; Meyers \& Greenberg, 1986).

People with early-onset depression tend to have more depressive cognitions, including suicidal thoughts, thoughts of worthlessness (Reinhard et al, 2000; Janssen et al, 2006) and neuroticism (Baldwin \& Tomenson, 1995; Van den Berg et al, 2001). Individuals with late-onset depression tend to present more with symptoms of psychomotor change, anhedonia, cognitive impairments (Hickie et al, 2001; Rapp et al, 2005) and weight loss (Janssen et al, 2006).

In contrast to these findings, a comparison study looking at early- and late-onset depression (onset after age 60) reported that the two groups were similar in terms of phenomenology, depressive subtype and severity, and neuropsychological performance (Brodaty et al, 2001). The validity of this study was perhaps affected by the fact that it was carried out in a tertiary referral centre and it might therefore reflect a more severe form of depression.

\section{Cognitive deficits}

Depression in all age groups is associated with some degree of impaired concentration and subjective difficulties with memory. Cognitive deficits seem to occur more frequently when first onset is in older age (Holroyd \& Duryee, 1997). Other cognitive deficits, such as episodic memory disturbance and executive dysfunction, are also commonly found in depression. Such deficits simply reflect a higher incidence of underlying physical morbidity in older patients as similar cognitive deficits occur in younger patients without physical comorbidity.

Memory improvement seems to correlate with amelioration of depressive signs and symptoms (Sternberg \& Jarvik, 1976). Yet in late-onset depression, cognitive deficits frequently persist, albeit to a lesser extent, following recovery from the episode, suggesting that they are not simply the result of depressed mood but instead are both 'state and trait' deficits (Nebes et al, 2003; Gallassi et al, 2006). There is also evidence that severity of cognitive deficits increases with severity of depression, regardless of age or education (Elderkin-Thompson et al, 2003). The magnitude of decline varies from just a trend to a statistically significant deficit, depending on the cognitive domain. This decline in cognitive performance also appears to run parallel to the trend seen in neuroanatomical studies, in which the volume of frontal and temporal lobes reduces with increasing severity of depression (see below).

Rapp et al (2005) measured neuropsychological function in 116 older adults (mean age just under 84 years) with late- or early-onset depression. They

Table 1 Clinical differences between late- and early-onset depression

\begin{tabular}{|c|c|c|}
\hline Source & Late-onset depression & Early-onset depression \\
\hline $\begin{array}{l}\text { Baldwin \& Tomenson, 1995; } \\
\text { Van den Berg et al, } 2001\end{array}$ & & Anxiety/neuroticism \\
\hline Janssen et al, 2006 & Weight loss & Anxiety, suicidal thoughts, worthlessness \\
\hline Reinhard et al, 2000 & & Low spirits, worthlessness \\
\hline Hickie et al, 2001 & $\begin{array}{l}\text { Cognitive impairment, anhedonia, } \\
\text { psychomotor changes }\end{array}$ & \\
\hline Rapp et al, 2005 & $\begin{array}{l}\text { Anhedonia; attentional and executive } \\
\text { dysfunction; cardiovascular comorbidity }\end{array}$ & Episodic memory dysfunction \\
\hline Brodaty et al, 2001 & $\begin{array}{l}\text { No difference in phenomenology or neurc } \\
\text { and early-onset }\end{array}$ & psychological performance between late- \\
\hline
\end{tabular}




\begin{tabular}{|c|c|c|}
\hline Source & Late-onset depression & Early-onset depression \\
\hline Maier et al, 1991 & & Higher rate of familial depression \\
\hline Brodaty et al, 2001 & & $\begin{array}{l}\text { Family history of any psychiatric illness, } \\
\text { personality abnormalities, history of poor } \\
\text { maternal relationships }\end{array}$ \\
\hline Mendlewicz \& Baron, 1981 & & $\begin{array}{l}\text { Greater family history of depression } \\
\text { (especially in female relatives), } \\
\text { alcoholism, sociopathy }\end{array}$ \\
\hline Van den Berg et al, 2001 & Severe life stress & Psychological vulnerability \\
\hline Grace \& O’Brien, 2003 & & $\begin{array}{l}\text { Bereavement life events, no difference on } \\
\text { Eysenck personality questionnaire }\end{array}$ \\
\hline Hein et al, 2003 & $\begin{array}{l}\text { Life time depressive symptoms and } \\
\text { somatic symptoms as preclinical markers }\end{array}$ & \\
\hline
\end{tabular}

found that individuals with late-onset depression had specific deficits in attention and executive function (consistent with frontal lobe dysfunction), whereas those with recurrent (early-onset) depression exhibited deficits in episodic memory (consistent with temporal lobe dysfunction). Sweeney et al (2000) identify the mesial temporal lobe as the area most associated with episodic memory.

\section{Late-onset depression and dementia}

When presented with symptoms of short-term memory impairment, psychomotor retardation, impaired concentration and apathy in elderly patients, clinicians are frequently faced with the challenge of differentiating between dementia and depression as the underlying diagnosis. Furthermore, a considerable number of elderly people develop late-onset depression alongside a dementing illness (Alexopoulos et al, 1993).

Kaimal \& Nair (2005) found studies reporting structural, functional and electrophysiological links between late-onset depression and Alzheimer's disease. It was suggested not that late-onset depression is a prodrome for any particular type of dementia, but that the majority of patients with late-onset depression who develop dementia get either Alzheimer's disease or vascular dementia. Several studies have looked at whether there is an association between apolipoprotein $\mathrm{E}$ and late-onset depression, but no association has been established (Hickie et al, 2001).

Van Reekum et al (1999) compared the cognitive status of 245 elderly individuals with either lateor early-onset depression and found that almost half of those with late-onset depression scored below the chosen cut-off for dementia, compared with just under one-third of those with early-onset depression. They also found that treatment of lateonset depression had little effect on cognition.

\section{Aetiology}

Various factors have been proposed as explanations for the onset of depression in early or later life (Table 2). These fall into the following broad categories.

\section{Genetic susceptibility}

Maier et al (1991) clearly established that people with late-onset depression (over 60 years of age at onset) have a higher familial rate of depression than age-matched controls, but the rate in early-onset depression is higher still. Brodaty et al (2001) found that a family history of any psychiatric illness is significantly more common in early- than in lateonset depression.

Mendlewicz \& Baron (1981) found that people with early-onset depression had a greater familial morbidity for depression, alcoholism and sociopathy than those with late-onset depression (onset after age 40). They also found an excess of depression in the female relatives of men and women with earlyonset depression compared with those with lateonset depression.

\section{Life events and psychosocial factors}

According to Van den Berg et al (2001), 'late-life depression' can be divided into subgroups with different aetiological pathways, including early onset with long-standing psychological vulnerability, and late onset as a reaction to severe life stress.

Hughes et al (1988) suggest that the frequency and severity of life events (e.g. loss of a spouse 
or physical illness) may be greater in later life in the general population. Grace \& O'Brien's (2003) findings that bereavement life events were more common in those with early-onset depression suggest that older people may be better able to cope with bereavements, even though they are at greater risk of experiencing them.

Brodaty et al (2001) found that personality abnormalities and a history of a poor maternal relationship were significantly more common in early- than in late-onset depression.

\section{Vascular disease}

In their 'vascular depression' hypothesis, Alexopoulos et al (1997) proposed that cerebral ischaemic damage to the fronto-subcortical circuits can predispose, precipitate and perpetuate late-onset depression. In support of their theory they cited a study that compared 21 patients with early-onset depression ( $<59$ years of age at onset) and 36 with late-onset depression (Baldwin \& Tomenson, 1995). Vascular risk factors were found to be highly significantly associated with late-onset depression, but not with just increasing age. This rules out ageing as a likely confounding association with vascular risk. The association between cardiovascular disease and depression is not confined to the elderly population, or those with severe depression.

Box 1 lists some of the theories proposed to explain the association between depression and vascular disease. Not all studies find an association between late-onset depression and vascular pathology. Janssen et al (2006) found no such association in an elderly (55-85 years of age) community-based sample. However, this may be explained by the

Box 1 Theories explaining the association between depression and vascular disease

- Increased platelet aggregation (Kempermann \& Kronenberg, 2003)

- Both depression and ischemia may be secondary to atherosclerosis (Baldwin \& O’Brien, 2002)

- Recurrent depression across the lifespan may increase the risk of vascular pathology (Baldwin \& O'Brien, 2002)

- Damagetoendarteriessupplyingsubcortical striato-pallido-thalamo-cortical pathways may disrupt the neurotransmitter circuitry involved in mood regulation, causing or predisposing to depression (Baldwin \& O’Brien, 2002) possibility that depression in a community sample is less likely to be complicated by factors such as comorbid vascular disease.

\section{Depressive symptoms/sub-threshold depression}

Hein et al (2003), in a 2-year prospective follow-up study, identified lifetime depressive and somatic symptoms as preclinical markers of late-onset depression. Certain symptoms, when present for over 2 weeks at any age, individually predicted lateonset depression. The most important of these were lack of joy and interest, poor concentration, increased appetite, lack of energy and joint pain. These findings suggest that previous sub-threshold depression increases the risk of later major depression.

\section{Structural neuroimaging}

Imaging studies in late-onset depression have found a reduction in grey matter volume in frontal (Krishnan, 1991) and temporal lobes, along with ventricular enlargement (Dahabra et al, 1998), sulcal widening, and reduction in volume of the caudate nucleus (Krishnan, 1991) and hippocampus (BellMcGinty et al, 2002).

Bell-McGinty et al (2002) found an inverse correlation between hippocampal-entorhinal volume and years since onset of depression, suggesting that depression may result in neuronal alterations at a molecular level in hippocampal cells. The glucocorticoid hypothesis suggests that hypercortisolaemia may damage hippocampal neurons, disrupting down-regulation of the hypothalamic-pituitary-adrenal (HPA) axis (Sapolsky et al, 1986). However, Lloyd et al (2004) found greater hippocampal atrophy in late-onset (after age 60) illness, contradicting the glucocorticoid hypothesis and suggesting that a pathological process other than exposure to hypercortisolaemia may underlie hippocampal atrophy. Candidate processes include oxidative stress, calcium metabolism and glutamatergic excitation.

\section{Prognosis}

Brodaty et al (1993) found that patients who experience their first episode of major depression before the age of 60 were more likely to have a relapsing course. This led them to conclude that early-onset depression predicts a poorer prognosis in elderly people than does late-onset depression. Asubsequent comparison study of individuals with early- and late-onset depression (Reynolds et al, 1998) found no difference between onset groups in the percentage 
of patients remitting, recovering or relapsing during their first year of maintenance treatment. However, people in the early-onset group tended to take 5-6 weeks longer to achieve remission.

Kalayam \& Alexopoulos (1999) found evidence that prefrontal dysfunction in elderly people is significantly associated with a poor or delayed response to antidepressants. In contrast, Navarro et al (2004) found that the presence of frontal hypoperfusion in individuals with late-onset depression was associated with quicker acute response to antidepressant treatment. However, they suggest that this reflects only the speed of response to antidepressants, and not remission rates.

Simpson et al (1998) studied the association between subcortical disease and antidepressant response in late-life depression, concluding that a poor response could be expected in patients with a 'vascular depression'. Miller et al (2002) found late-onset depression (at over 60 years of age) was associated with cerebrovascular risk factors. Yet, a high risk of cerebrovascular disease and onset of depression after the age of 60 had no effect on time to remission, need for adjunctive treatment or risk of recurrence over 3-year follow-up.

\section{Management options}

In the assessment of patients presenting with depression occurring for the first time in late life it is particularly important to consider neurological and vascular factors, examples of which are listed in Box 2. Baldwin et al's (2005) report of increased neurological findings in late-onset depression emphasises the importance of physical examination and thorough assessment of cognition. Since vascular disease has been found to be associated with lateonset depression, identification and modification of risk factors for vascular disease, including

Box 2 Vascular and atrophic changes in lateonset depression

- Ischaemia (Baldwin \& Tomenson, 1995; Alexopoulos et al, 1997)

- Ventricular enlargement (Alexopoulos et al, 1993)

- Electrocardiogram (EEG) changes (Kaimal \& Nair, 2005)

- Reduction in grey matter in frontal lobe and caudate nucleus (Krishnan, 1991)

- Reduction in volume of hippocampus (BellMcGinty et al, 2002; Baldwin et al, 2004; Hickie et al, 2005) hypertension, raised cholesterol, diabetes and smoking, plays an important role in prevention and management of depression.

People with late-onset depression have a higher propensity for developing cognitive deficits than those with early-onset depression (Alexopoulos, et al, 1997), making them more vulnerable to cognitive side-effects of medications, including psychotropics, and electroconvulsive therapy (ECT). In the latter, unilateral ECT, a low-dosing electrical stimulus and adequate pre-anaesthetic may be used to reduce the risk of cognitive side-effects. Evidence suggests that ECT can result in persistent or permanent loss of explicit memory and retrograde amnesia (Sackheim, 2000; American Psychiatric Association, 2001). A proportion of patients continue to complain of subjective memory problems following ECT even when no objective evidence is found on formal testing. However, identifying and monitoring cognitive symptoms may be particularly important in this vulnerable group. Robertson \& Pryor (2006) suggest the use of various neuropsychological batteries for testing cognitive function following ECT.

We were unable to find any evidence supporting or refuting the use of psychotherapies in late-onset depression. The National Institute for Health and Clinical Excellence (NICE) guidelines for depression recommend that people over the age of 65 should be offered a full range of psychological treatments, including cognitive-behavioural therapy and supportive counselling (National Collaborating Centre for Mental Health, 2004).

We were unable to find any evidence for the use of antioxidants (vitamin E, fish oils) in the management of cortisol-induced hippocampal damage.

For a general review of the management of depression in later life readers are referred to Baldwin \& Wild's (2004) article in this journal.

\section{Conclusions}

Clinicians should recognise that late-onset depression differs from the early-onset disorder. Research suggests that it is more difficult to diagnose and is more commonly associated with cognitive impairments that complicate management. An increase in the occurrence of vascular disease in elderly people may complicate the clinical picture: cardiovascular risk factors require optimum management in older people with the late-onset depression. Despite some evidence that depression of late onset has a poorer outcome than the earlyonset disorder, we were unable to confirm this, as studies provide conflicting results.

Defining age at onset of a depression can be difficult; symptoms may have been present for some time but 
not recognised as being part of a depressive illness. Memory problems associated with depression may affect reliability of recall of symptom onset. Residual features of previous physical illness or depression can affect the clinical picture in subsequent episodes of early-onset depression, thus complicating attempts to differentiate it from depression of late onset.

This review has highlighted the paucity of research evidence on the pathophysiology, prognosis and management of late-onset depression. Study of the functional outcomes and underlying pathophysiological mechanisms of this disorder might inform the development of care pathways involving compensatory coping strategies.

\section{Declaration of interest}

None.

\section{Acknowledgement}

We thank Drs D. Yadav and S. Mukunda for their help in the literature search.

\section{References}

Alexopoulos, G. S., Meyers, B. S., Young, R. C., et al (1993) The course of geriatric depression with reversible dementia: a controlled study. American Journal of Psychiatry, 150, 1693-1699.

Alexopoulos, G. S., Meyers, B. S., Young, R. C., et al (1997) 'Vascular depression' hypothesis. Archives of General Psychiatry, 54, 915-922.

American Psychiatric Association (2001) ECT Task Force of the American Psychiatric Association: The Practice of Electroconvulsive Therapy. American Psychiatric Press.

Ayuso-Mateos, J. L., Vázque-Barquero, J. L., Dowrick, C., et al (2001) Depressive disorders in Europe: prevalence figures from the ODIN study. British Journal of Psychiatry, 179, 308-316.

Baldwin, R. C. \& O'Brien, J. (2002) Vascular basis of lateonset depressive disorder. British Journal of Psychiatry, 180, 157-160.

Baldwin, R. C. \& Tomenson, B. (1995) Depression in later life. A comparison of symptoms and risk factors in early and late onset cases. British Journal of Psychiatry, 167, 649-652.

Baldwin, R. \& Wild, R. (2004) Management of depression in later life. Advances in Psychiatric Treatment, 10, 131-139.

Baldwin, R., Jeffries, S., Jackson, A., et al (2004) Treatment response in late-onset depression: relationship to neuropsychological, neuroradiological and vascular risk factors. Psychological Medicine, 34, 125-136.

Baldwin, R., Jeffries, S., Jackson, A., et al (2005) Neurological findings in late-onset depressive disorder: comparison of individuals with and without depression. British Journal of Psychiatry, 186, 308-313.

Bellini, M. \& Matteucci, V. (2001) Late onset depression and suicide outcome. Archives of Gerontology and Geriatrics, 33, 37-42.

Bell-McGinty, S., Butters, M. A., Meltzer, C .C., et al (2002) Brain morphometric abnormalities in geriatric depression: long-term neurobiological effects of illness duration. American Journal of Psychiatry, 159, 1424-1427.

Brodaty, H., Harris, L., Peters, K., et al (1993) Prognosis of depression in the elderly. A comparison with younger patients. British Journal of Psychiatry, 163, 589-596.

Brodaty, H., Luscombe, G., Gordon, P., et al (2001) Early and late onset depression in old age. Different aetiologies, same phenomenology. Journal of Affective Disorders, 66, 225-236.
Caine, E. D., Lyness, J. M. \& King, D. A. (1994) Clinical and etiological heterogeneity of mood disorders in elderly patients. In Diagnosis and Treatment of Depression in Late Life: Results of the NIH Consensus Development Conference (eds S. Lon, M. D. Schneider, C. F. Reynolds, et al) pp. 23-53. American Psychiatric Press.

Dahabra, S., Ashton, H. C., Bahrainain M., et al (1998) Structural and functional abnormalities in elderly patients clinically recovered from early- and late-onset depression. Biological Psychiatry, 44, 34-46.

Elderkin-Thompson, V., Kumar, A., Bilker, W. B., et al (2003) Neuropsychological deficits among patients with lateonset minor and major depression. Archives of Clinical Neuropsychology, 18, 529-549.

Fountoulakis, K. N., O'Hara, R., Iacovides, A., et al (2003) Unipolar late-onset depression. Acomprehensive review. Annals of General Hospital Psychiatry, 2, 11 (doi: 10.1186/1475-2832-2-11).

Gallassi, R., Di Sarro, R., Morreale, A., et al (2006) Memory impairment in patients with late-onset major depression: the effect of antidepressant therapy. Journal of Affective Disorders, 91, 243-250.

Grace, J. \& O'Brien, J. T. (2003) Association of life events and psychosocial factors with early but not late onset depression in the elderly: implications for possible differences in aetiology. International Journal of Geriatric Psychiatry, 18, 473-478.

Gurland, B. J. (1976) The comparative frequency of depression in various adult age groups. Journal of Gerontology, 31, 283-292.

Hein, S., Bonsignore, M., Barkow, K., et al (2003) Lifetime depressive and somatic symptoms as preclinical markers of late-onset depression. European Archives of Psychiatry and Clinical Neuroscience, 253, 16-21.

Hickie, I., Scott, E., Naismith, S., et al (2001) Late-onset depression: genetic, vascular and clinical contributions. Psychological Medicine, 31, 1403-1412.

Hickie, I., Naismith, S., Ward, P. B., et al (2005) Reduced hippocampal volumes and memory loss in patients with earlyand late-onset depression. British Journal of Psychiatry, 186, 197-202.

Holroyd, S. \& Duryee, J. J. (1997) Differences in geriatric psychiatry outpatients with early- vs late-onset depression. International Journal of Geriatric Psychiatry, 12, 1100-1106.

Hopkinson, G. (1964) A genetic study of affective illness in patients over 50. British Journal of Psychiatry, 110, 244-254.

Hughes, D. C., Blazer, D. G. \& George, L. K. (1988) Age differences in life events: a multivariate controlled analysis. International Journal of Aging and Human Development, 27, 207-220.

Janssen, J., Beekman, A. T. F., Comijs, H. C., et al (2006) Latelife depression: the differences between early- and late-onset illness in a community-based sample. International Journal of Geriatric Psychiatry, 21, 86-93.

Kaimal, A. B. \& Nair, U. V. (2005) Organic brain dysfunction in late-onset depression. British Journal of Psychiatry, 187, 288.

Kalayam, B. \& Alexopoulos, G. S. (1999) Prefrontal dysfunction and treatment response in geriatric depression. Archives of General Psychiatry, 56, 713-718.

Kempermann, G. \& Kronenberg, G. (2003) Depressed new neurons: adult hippocampal neurogenesis and a cellular hypothesis of major depression. Biological Psychiatry, 54, 499-503.

Krishnan, K. R. R. (1991) Organic basis of depression in the elderly. Annual Review of Medicine, 42, 261-266.

Lloyd, A. J., Ferrier, I. N., Barber, R., et al (2004) Hippocampal volume change in depression: late- and early-onset illness compared. British Journal of Psychiatry, 184, 488-495.

Lockwood, K. A., Alexopoulos, G. S., Kakuma, T., et al (2000) Subtypes of cognitive impairment in depressed older adults. American Journal of Geriatric Psychiatry, 8, 3.

Maier, W., Litchtermann, D., Heun, R., et al (1991) Unipolar depression in the aged. Determinants of familial aggregation. Journal of Affective Disorders, 23, 53-61.

Mendlewicz, J. \& Baron, M. (1981) Morbidity risks in subtypes of unipolar depressive illness: differences between early and late onset forms. British Journal of Psychiatry, 139, 463-466.

Meyers, B. S. \& Greenberg, R. (1986) Late-life delusional depression. Journal of Affective Disorders, 11, 133-137.

Miller, M. D., Lenze, E. J., Dew, M. A., et al (2002) Effect of cerebrovascular risk factors on depression treatment outcome 
in later life. American Journal of Geriatric Psychiatry, 10, 592-598.

National Collaborating Centre for Mental Health (2004) Depression: Management of Depression in Primary and Secondary Care. Clinical Guideline 23. NICE (http://guidance.nice.org. $\mathrm{uk} / \mathrm{CG} 23 /$ ? $\mathrm{c}=91523$ ).

Navarro, V., Gasto, C., Lomena F., et al (2004) Prognostic value of frontal functional neuroimaging in late-onset severe major depression. British Journal of Psychiatry, 184, 306-311.

Nebes, R., Pollock, B. G., Houck, P. R., et al (2003) Persistence of cognitive impairment in geriatric patients following antidepressant treatment: a randomised, double-blind clinical trail with nortriptyline and paroxetine. Journal of Psychiatric Research, 37, 99-108.

Rapp, M. A., Dahlman, K., Sano, M., et al (2005) Neuropsychological differences between late-onset and recurrent geriatric major depression. American Journal of Psychiatry, 162, 691-698.

Reinhard, H., Martin, K. \& Andreas P., (2000) Distinction of early-and late-onset depression in the elderly by their lifetime symptomatology. International Journal of Geriatric Psychiatry, 15, 1138-1142.

Reynolds, C. F., Dew, M. A. \& Frank, E. ( 1998) Effects of age at onset of first lifetime episode of recurrent major depression on treatment response and illness course in elderly patients. American Journal of Psychiatry, 155, 795-799.

Robertson, H. \& Pryor, R. (2006) Memory and cognitive effects of ECT: informing and assessing patients. Advances in Psychiatric Treatment, 12, 228-238.

Sackheim, H. A. (2000) Memory and ECT: from polarization to reconciliation. Journal of ECT, 16, 87-97.

Sapolsky, R. M., Krey, L. C. \& McEwen, B. S. (1986) The neuroendocrinology of stress and aging: the glucocorticoid cascade hypothesis. Endocrine Reviews, 7, 284-301.

Simpson, S., Baldwin, R. C., Jackson, A., et al (1998) Is subcortical disease associated with a poor response to antidepressants? Neurological, neuropsychological and neuroradiological findings in late-life depression. Psychological Medicine, 28, 1015-1026.

Sternberg, D. F. \& Jarvik, M. E., (1976) Memory functions in depression. Archives of General Psychiatry. 33, 219-224.

Sweeney, J. A., Kmiec, J. A. \& Kupfer, D. J. (2000) Neuropsychologic impairments in bipolar and unipolar mood disorders on the CANTAB neurocognitive battery. Biological Psychiatry, $48,674-684$.

Van den Berg, M. D., Oldehinkel, A. J., Bouhuys, A. L., et al (2001) Depression in later life. Three etiological different subgroups. Journal of Affective Disorders, 65, 19-26.

van Reekum, R., Simard, M., Clarke, D., et al (1999) Late-life depression as a possible predictor of dementia, cross-sectional and short-term follow-up results. American Journal of Geriatric Psychiatry, 7, 151-159.

\section{MCQs}

1 Compared with early-onset depression, late-onset depression is associated with:

a lower suicide rates

b less treatment resistance

c lower incidence of hypochondriasis

d higher neuroticism

e cognitive impairment.
2 Compared with late-onset depression, early-onset depression is more associated with:

a frontal lobe dysfunction

b attentional problems

c executive dysfunction

d episodic memory dysfunction

e temporal lobe dysfunction.

3 In dementia:

a depression may mimic its symptoms and signs

b late-onset depression is not associated with APOE e4

c depression is not a risk factor

d late-onset depression is always a prodrome of Alzheimer's disease

e late-onset depression is a prodrome of vascular dementia.

4 In terms of aetiology, early-onset depression can be more associated than late-onset depression with:

a family history

b vascular disease

c reduced hippocampal volume

d smaller prefrontal lobe volume

e smaller caudate nuclear volume.

5 It is true to say that:

a early-onset depression always has a better outcome than late-onset depression

b oxidative stress leads to neuronal cell death

c ECT is not associated with irreversible memory problems

d it is not possible to clinically monitor cognitive effects of ECT

e late-onset depression is not associated with vascular dementia.

\section{MCQ answers}

\begin{tabular}{|c|c|c|c|c|}
\hline 1 & 2 & 3 & 4 & 5 \\
\hline a $F$ & a $F$ & a $\mathrm{T}$ & a $\mathrm{T}$ & a $F$ \\
\hline b F & b F & b F & b F & b $\mathrm{T}$ \\
\hline c F & c F & C F & c F & C $\mathrm{F}$ \\
\hline d F & d $\mathrm{T}$ & d F & d F & d F \\
\hline e $\mathrm{T}$ & e $F$ & e F & e $F$ & e $F$ \\
\hline
\end{tabular}

